\title{
A generative model for constructing nucleic acid sequences binding to a protein
}

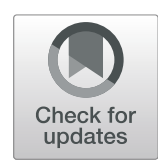

Jinho $\mathrm{Im}^{\dagger}$, Byungkyu Park ${ }^{\dagger}$ and Kyungsook $\mathrm{Han}^{*}$

From 2018 International Conference on Intelligent Computing (ICIC 2018) and Intelligent Computing and Biomedical Informatics (ICBI) 2018 conference

Wuhan and Shanghai, China. 15-18 August 2018, 3-4 November 2018

\begin{abstract}
Background: Interactions between protein and nucleic acid molecules are essential to a variety of cellular processes. A large amount of interaction data generated by high-throughput technologies have triggered the development of several computational methods either to predict binding sites in a sequence or to determine whether a pair of sequences interacts or not. Most of these methods treat the problem of the interaction of nucleic acids with proteins as a classification problem rather than a generation problem.

Results: We developed a generative model for constructing single-stranded nucleic acids binding to a target protein using a long short-term memory (LSTM) neural network. Experimental results of the generative model are promising in the sense that DNA and RNA sequences generated by the model for several target proteins show high specificity and that motifs present in the generated sequences are similar to known protein-binding motifs.

Conclusions: Although these are preliminary results of our ongoing research, our approach can be used to generate nucleic acid sequences binding to a target protein. In particular, it will help design efficient in vitro experiments by constructing an initial pool of potential aptamers that bind to a target protein with high affinity and specificity.
\end{abstract}

Keywords: Aptamer, Protein-nucleic acid binding, Recurrent neural network

\section{Introduction}

Due to recent advances in high-throughput experimental technologies, a large amount of data on interactions between proteins and nucleic acids have been generated. Motivated by the increased amount of data on proteinnucleic acid interactions, several machine learning methods have been used either to predict binding sites in a sequence [1-4] or to determine if an interaction exists between a pair of sequences [5-9].

Among the machine learning methods, variants of neural networks were applied to predict the interactions between proteins and nucleic acids. For example, DeepBind [5] is a convolutional neural network trained on a huge amount of data from high-throughput experimental

\footnotetext{
*Correspondence: khan@inha.ac.kr

† Jinho Im and Byungkyu Park contributed equally to this work.

'Department of Computer Engineering, Inha University, 22212 Incheon, South Korea
}

technologies. For the problem of predicting proteinbinding sites of nucleic acid sequences, DeepBind contains hundreds of distinct prediction models, each for a different target protein. As output, it provides a predictive binding score without suggesting protein-binding sites in the input nucleic acid sequence. Nonetheless, it provides informative predictions for many target proteins, so we used DeepBind to estimate the affinity and specificity of nucleic acid sequences generated by our model for a target protein.

A more recent model called DeeperBind [10] predicts the protein-binding specificity of DNA sequences using a long short-term recurrent convolutional network. By employing more complex and deeper layers, DeeperBind showed a better performance than DeepBind for some proteins, but its use is limited to the datasets from proteinbinding microarrays. Both DeepBind and DeeperBind are classification models rather generative models, so cannot 
be used to construct nucleic acid sequences that potentially bind to a target protein.

There are a few computational methods that generate protein-binding nucleic acid sequences. Most of them include two steps: generating candidate sequences and testing the sequences. For instance, Kim et al. [11] generated a large number of RNA sequences using nucleotide transition probability matrices and selected candidate sequences with specified secondary structures and motifs. Their approach is quite exhaustive and requires a large amount of computational power. Zhou et al. [12] generated RNA sequences that can form a desired RNA motif, and selected potent aptamers by molecular dynamics simulation-based virtual screening. Hoinka et al. [13] developed a program called AptaSim for simulating the selection dynamics of HT-SELEX experiments based on a Markov model.

The main difference of our approach from the others is that our approach is a deep learning model that can be trained directly on data from high-throughput experiments such as HT-SELEX or CLIP-seq. After being trained on experimental data, our model generates sequences similar to those in a training dataset, and evaluates the sequences with respect to binding affinity and specificity to a target protein. A limitation of our model is that it requires experimental data for training and a classifier of protein-binding nucleic acids. However, this limitation is expected to be overcome in the near future as a large amount of experimental data is being generated through high-throughput experiments.

This paper presents a generative model that constructs potential aptamers for a target protein. Aptamers are synthetic but biologically active, short single-stranded nucleic molecules which bind to a target molecule with high affinity and specificity [14]. The preliminary results show that our approach can generate nucleic acid sequences that bind to a target protein with high affinity and specificity, which will definitely help design in vitro or in vivo experiments to finalize aptamers for target proteins. To the best of our knowledge, this is the first attempt to generate potential aptamers using a recurrent neural network.

\section{Materials and methods}

\section{Data set}

The data set used for training the generator model was obtained from the DeepBind site at http://tools.genes. toronto.edu/deepbind/nbtcode. The data set includes a large number DNA sequences binding to one of 396 transcription factors (TFs). In the data set, 20-mer DNA sequences bind to most TFs (320 out of 396 TFs), 14-mer DNA sequences bind to $14 \mathrm{TFs}$, and 40-mer DNA sequences bind to $25 \mathrm{TFs}$. Thus, we selected the most typical length of 20 as the length of DNA sequences generated by our model.
In the data set, setA contains positive data (i.e., proteinbinding DNA sequences) and setB contains negative data (i.e., non-binding DNA sequences). We used setA to train our generator model. For comparison of our method with others, the HT-SELEX data was obtained from https:// www.ncbi.nlm.nih.gov/bioproject/371436. Both data sets are also available in Additional file 1.

\section{Sequence generator}

A recurrent neural network (RNN) is capable of learning the property of sequential data such as time series data or text data. However, RNN suffers from the vanishing gradient problem, in which the gradients vanish and consequentially the parameters are not updated during back propagation. Long short-term memory (LSTM) solves the vanishing gradient problem of RNN by introducing a gating mechanism [15]. LSTM allows the network to determine when and what to remember or forget. LSTM has shown a great performance in speech recognition [16] and language translation [17].

We implemented a generator model of nucleic acid sequences using char-rnn (https://github.com/karpathy/ char-rnn). Our model is composed of two layers of LSTM with 128 hidden neurons (Fig. 1). Given a sequence of characters, it reads one character of the sequence at a time and predicts the next in the sequence.

In the LSTM model, the batch size (B) specifies how many streams of data are processed in parallel at one time. The sequence length $(S)$ specifies the length of each stream $(S=20$ in our dataset). Suppose that an input file to a model has $\mathrm{k}$ DNA sequences of 20 nucleotides and that $N=k \times 20$. Then, the input file of $\mathrm{N}$ characters is split into data chunks of size $B \times 20$. By default, $95 \%$ of the data chunks are used for training and $5 \%$ of the chunks are used to estimate the validation loss. The input file is split into data chunks and fed to the LSTM layers with default settings. In our study, we used the default value of 50 for the batch size (B).

The LSTM model was trained in the following way (Eq. 1). Let $x_{t}$ be a vector representing the $t$-th nucleotide in the input sequence. Only one element of $x_{t}$ is 1 and the others are 0. $y_{t}$ is a class indicator of $n_{t}$ defined by Eq. 1 . The LSTM calculates $z_{t}$ for $x_{t}$ (Eq. 2). Softmax changes $z_{t}$ to a vector of values between 0 and 1 that sum to 1 , and softmax $j$ is the $j$-th element of the output of the softmax (Eq. 3). The loss is the mean of the negative log-likelihood of the prediction (Eq. 4). The loss is used to update the hidden neurons in the hidden layer using the RMSProp algorithm [18]. When generating a sequence, the model takes a vector $(0.25,0.25,0.25,0.25)$ as $x_{1}$ and computes $\operatorname{softmax}\left(z_{t}\right)$, a multinomial distribution of nucleotides. One character is sampled from the distribution and the vector of the character fed back to the model as $x_{2}$. This process is 
repeated until it reaches the pre-determined length of the sequence.

$$
\begin{aligned}
& x_{t}=4 \text {-bit number representing a nucleotide } \\
& n_{t} \in\{A, C, G, T(U)\} \\
& y_{t}=\left\{\begin{array}{l}
1 \text { if } n_{t}=A \\
2 \text { if } n_{t}=C \\
3 \text { if } n_{t}=G \\
4 \text { if } n_{t}=T(U)
\end{array}\right. \\
& z_{t}=\operatorname{LSTM}\left(x_{t}\right) \\
& \operatorname{softmax}_{j}\left(z_{t}\right)=e^{z_{t j}} / \sum_{k=1}^{4} e^{z_{t k}}, j \in\{1,2,3,4\} \\
& \text { loss }=-\sum_{t=1}^{|x|} \ln \left(\operatorname{softmax}_{y_{t}}\left(z_{t}\right)\right) /|x|
\end{aligned}
$$

For protein-binding DNA sequences, the model was trained on a set of DNA sequences, which were identified by HT-SELEX experiments as binding sequences to human transcription factors [19]. Among the transcription factors, we selected those with a known aptamer. Since the DNA sequences used in training the model were 20 nucleotide long, the length of nucleic acid sequences generated by the model was also set to 20 nucleotides. When training the model, the results were evaluated with respect to two measures: loss and intersection to union (IU) ratio, which are defined by Eqs. 4 and 5, respectively.

$\mathrm{IU}$ ratio $=\frac{\{\text { training sequences }\} \cap\{\text { generated sequences }\}}{\{\text { training sequences }\} \cup\{\text { generated sequences }\}}$

Figure 2 shows the IU ratios and loss values of the model during the first 50 epochs of training for NFATC1 and NFKB1. For both NFATC1 and NFKB1, the IU ratio was increased as the model was trained longer (Fig. 2a). In contrast to the IU ratio, the loss tended to be decreased after a certain point as the model was trained longer, but the decreasing trend was not monotonic. The loss of the model for NFKB1 converged to $\sim 1.05$, whereas that for NFATC1 was increased slightly after reaching to the minimum loss of 0.95 at epoch 19.

The model with the maximum IU ratio generated many redundant sequences. About 25\% and 33\% of the sequences generated by the model for NFKB1 and NFATC1 were duplicated sequences, respectively. Thus, we selected a generator model with the minimum loss

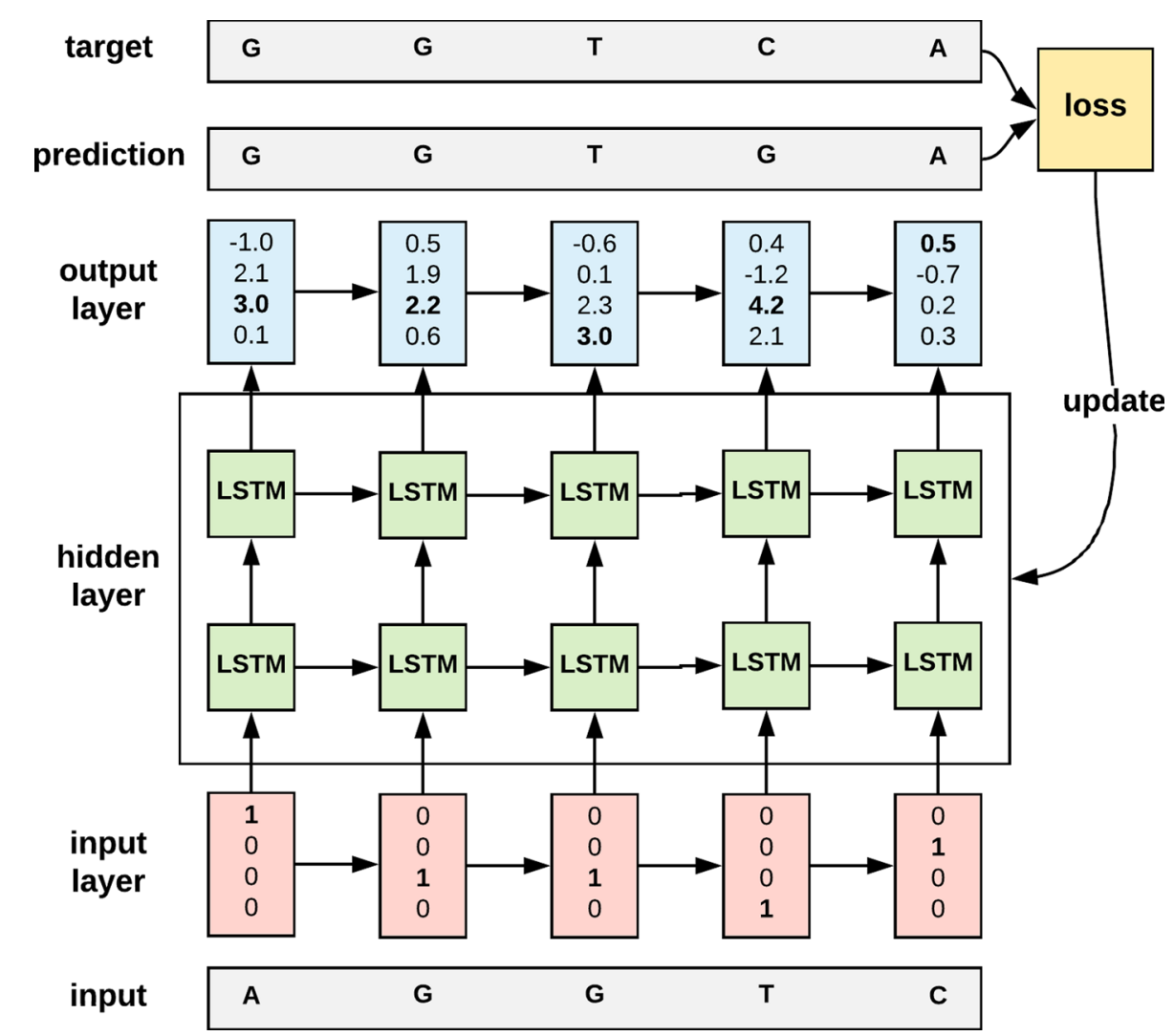

Fig. 1 The architecture of the sequence generator. The loss is used to update the hidden neurons in the hidden layer using the RMSProp algorithm [18] 


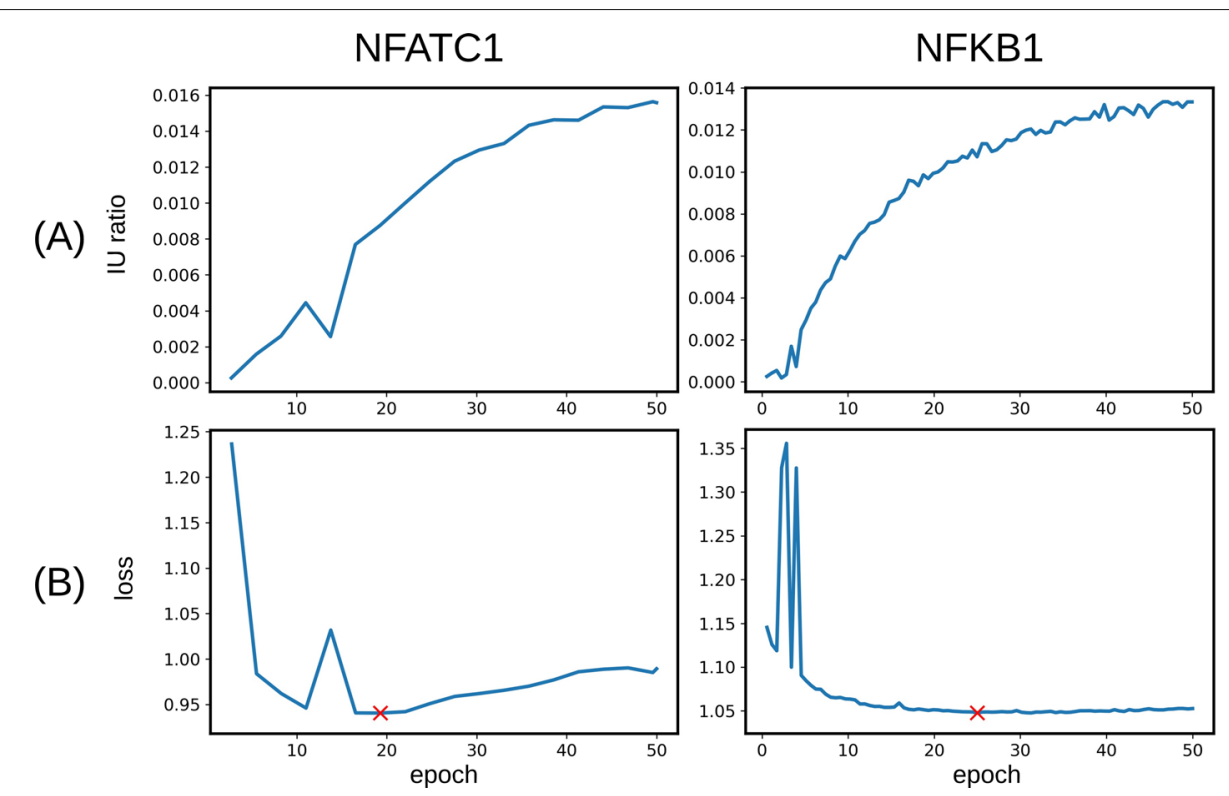

Fig. 2 a The IU ratio of the model during the first 50 epochs of training for NFATC1 and NFKB1. $\mathbf{b}$ The loss of the model during the first 50 epochs of training. The red symbol ' $x$ ' represents the minimum loss point

value rather than one with the maximum IU ratio to construct various sequences which are similar, but not exactly the same, to those in the training set.

\section{Binding affinity and specificity}

To evaluate the binding affinity and specificity of nucleic acid sequences to a target protein, we used the predictive binding score of DeepBind (hereafter called DeepBind score) [5]. Figure 3 shows DeepBind scores of random sequences in 10 DeepBind models. As shown in Fig. 3, the scale of DeepBind scores is arbitrary, thus DeepBind scores from different DeepBind models are not directly comparable.

To make DeepBind scores comparable, we defined the binding affinity $(A F)$ of a nucleic acid sequence $s$ to a target protein $p$ as the probability that the DeepBind score of $s$ would be higher than that of a random sequence. To obtain an approximate value of the probability, we ran DeepBind on 200,000 random DNA sequences of 20 nucleotides and computed their binding affinity by Eq. 6 . Since the binding affinity is a probability, it is always in the range of $[0,1]$. In the equation, $\operatorname{Score}_{m}(s)$ and $\operatorname{Score}_{m}\left(r_{i}\right)$ represent the score of a sequence $s$ and the score of the $i$-th random sequence, respectively, computed by DeepBind model $m$. The procedure for computing the binding affinity is illustrated in Fig. 4.

$A F_{p}(s)=\frac{1}{n} \sum_{i=1}^{n} \delta\left(\operatorname{Score}_{m}(s) \geq \operatorname{Score}_{m}\left(r_{i}\right)\right)$

where $\delta(A)=1$ if an event A occurs; $\delta(A)=0$ otherwise.

Table 1 shows some positive data used for training and testing DeepBind models for several target proteins along

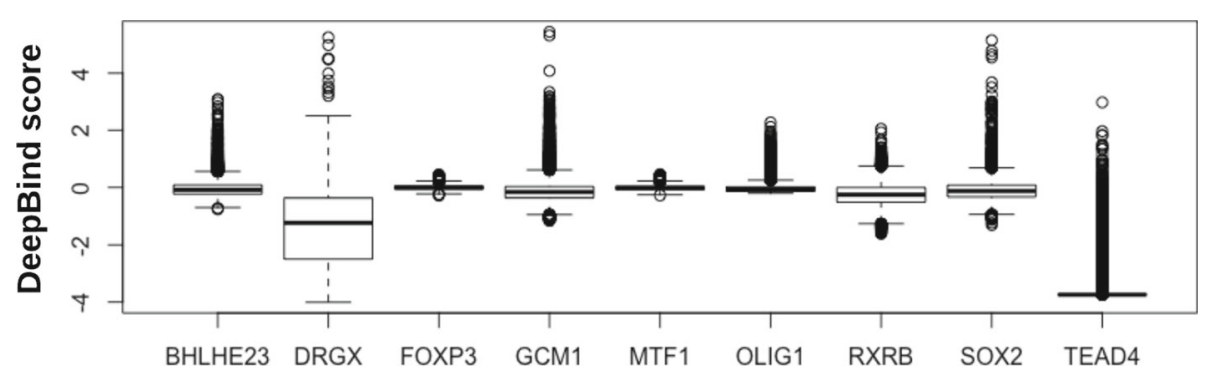

Fig. 3 DeepBind scores of random sequences, calculated by 9 DeepBind models for 9 proteins (BHLHE23, DRGX, FOXP3, GCM1, MTF1, OLIG1, RXRB, SOX2, and TEAD4) 


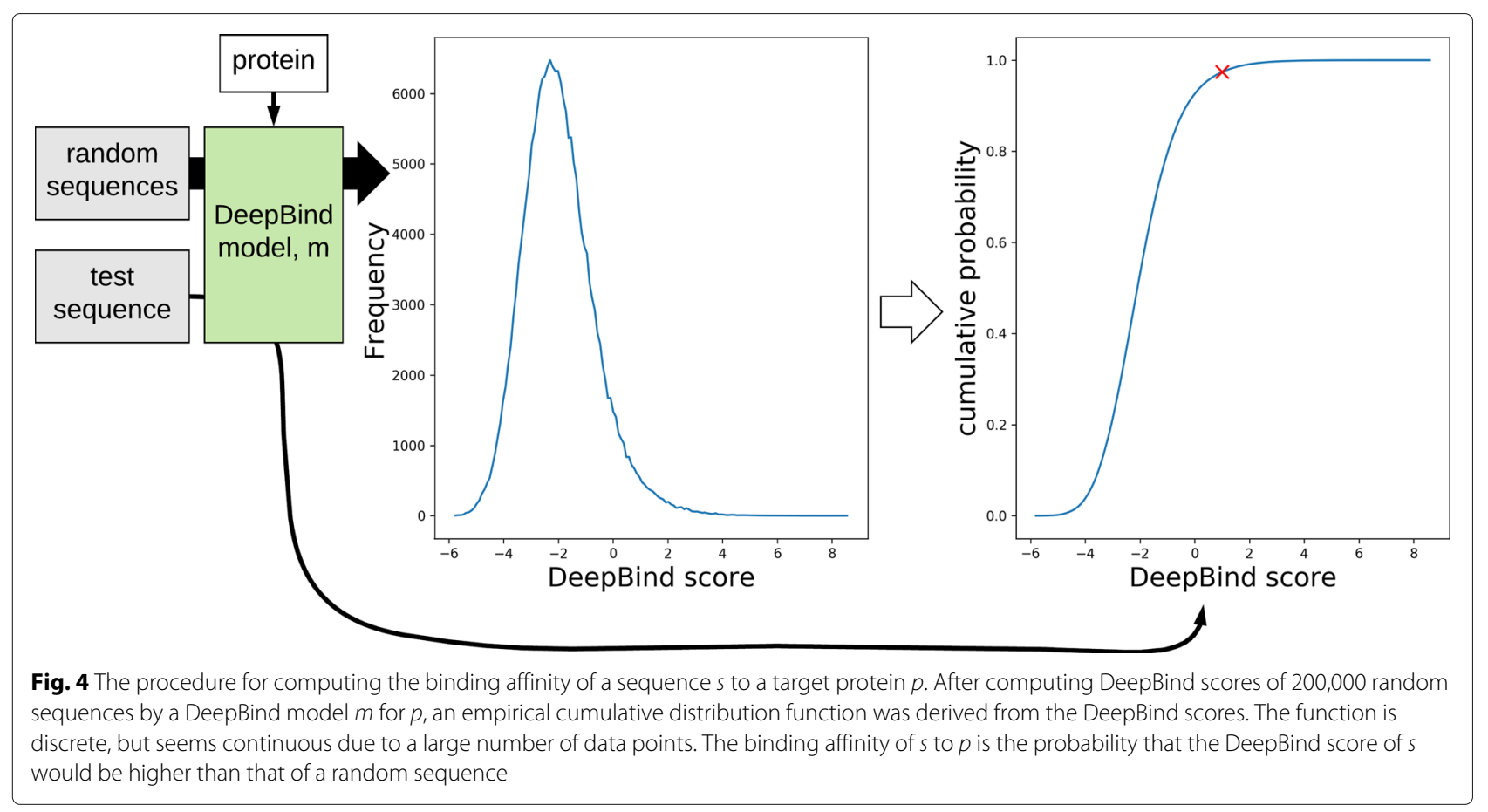

with AUC values in testing. Different DeepBind models show very different AUC values, ranging from 0.499 for FOXP3 to 0.990 for TEAD4. The AUC value of 0.499 in testing is close to random guessing.

We defined the binding specificity $(S P)$ of a nucleic acid sequence $s$ to a target protein $p$ by Eq. 7. The binding specificity of $s$ to $p$ is the difference between the AUCweighted binding affinity $A F$ of $s$ to $p$ and the AUCweighted mean $A F$ of $s$ to all other proteins except $p$. In the equation, $M$ is a set of all generator models trained on data from the same type of experiment as $m$. The binding affinity $A F$ is weighted by AUC to reflect the reliability of each model. When the AUC value is not available, $A F$ is not weighted by AUC (i.e., $A U C_{m}=1$ for every model $m$ ).

$$
\begin{aligned}
& M^{c}=M-\{m\} \\
& S P_{p}(s)=A F_{p}(s) \cdot A U C_{m}-\frac{1}{\left|M^{c}\right|} \sum_{k \in M^{c}} A F_{k}(s) \cdot A U C_{k}
\end{aligned}
$$

\section{Algorithm}

To construct potential aptamers for a protein target, our model requires three inputs: a target protein, a training

\begin{tabular}{|c|c|c|c|c|c|}
\hline Protein & Type & Species & Family & Experiment & AUC in test data \\
\hline TEAD4 & TF & H. sapiens & TEA & ChIP-seq & 0.990 \\
\hline NFATC1 & TF & H. sapiens & Rel & SELEX & 0.909 \\
\hline DRGX & TF & H. sapiens & Homeodomain & SELEX & 0.897 \\
\hline GCM1 & TF & H. sapiens & GCM & SELEX & 0.841 \\
\hline NFKB1 & TF & H. sapiens & Rel & SELEX & 0.771 \\
\hline OLIG1 & TF & H. sapiens & bHLH & SELEX & 0.733 \\
\hline RXRB & TF & H. sapiens & Nuclear receptor & SELEX & 0.720 \\
\hline SOX2 & TF & H. sapiens & Sox & SELEX & 0.605 \\
\hline BHLHE23 & TF & H. sapiens & bHLH & SELEX & 0.557 \\
\hline MTF1 & TF & H. sapiens & $\mathrm{C} 2 \mathrm{H} 2 \mathrm{ZF}$ & SELEX & 0.538 \\
\hline FOXP3 & TF & H. sapiens & Forkhead & SELEX & 0.499 \\
\hline MBNL1 & RBP & H. sapiens & Znf & RNAcompete & - \\
\hline
\end{tabular}

Table 1 Part of positive data from [19] used for training and testing DeepBind

Negative data was prepared by shuffling dinucleotides. TF: transcription factor. RBP: RNA binding protein. AUC of DeepBind for MBNL1 is not available 
set of nucleic acid sequences binding to the target protein, and a set of DeepBind models. A DeepBind model for the target protein should be included in the input. After training the model on the training dataset for 50 epochs, we select a model with the lowest loss value. The selected model is used to generate nucleic acid sequences, and the binding affinity and specificity of the generated sequences to the target protein are computed using Eq. 3 and 4, and the top 100 sequences with the highest binding specificity are chosen as potential aptamers of the target protein. A high-level description of our approach is outlined in Algorithm 1.

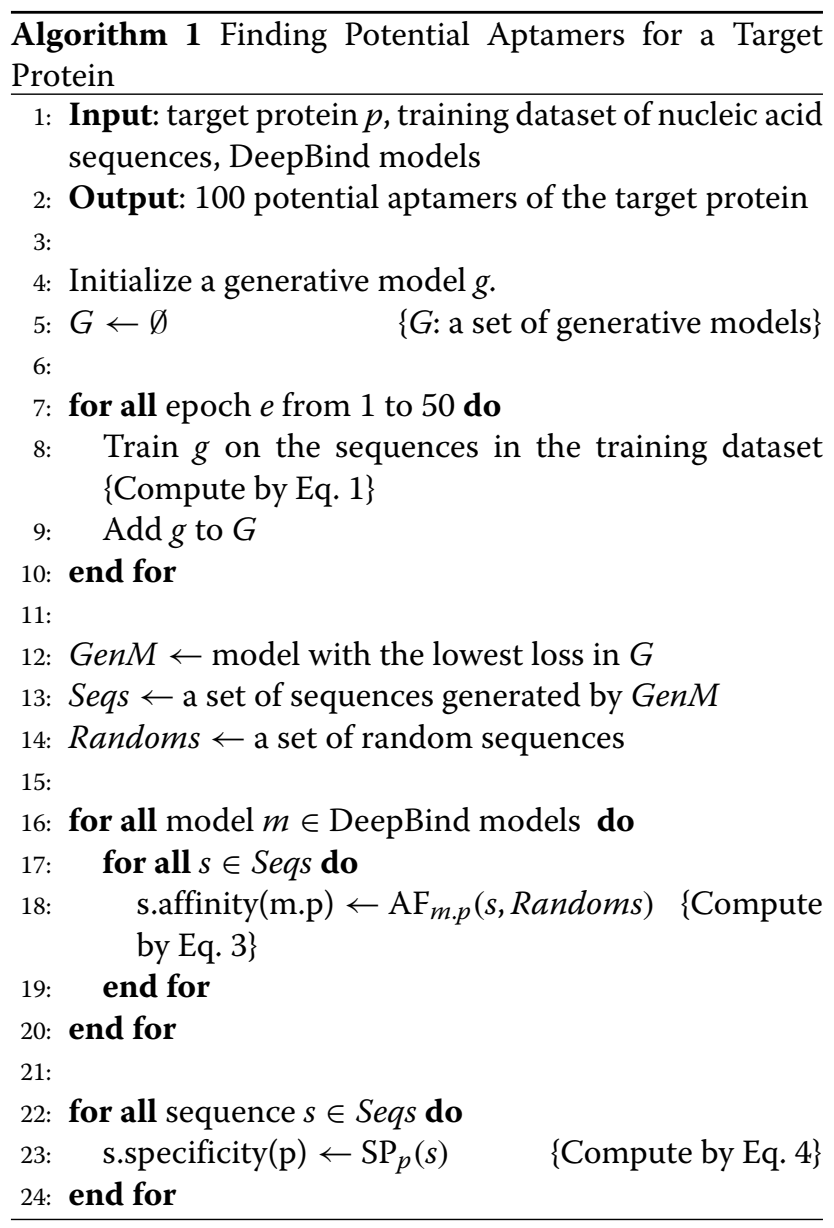

\section{Results and discussion} Binding affinity of generated sequences

To examine the protein-binding affinity of DNA sequences, we generated DNA sequences binding to several proteins shown in Table 1. For each target protein, Table 2 shows AUC of the protein's DeepBind model and median protein-binding affinity $A F$ of the generated sequences and random sequences. For comparison we used the median $A F$ value instead of the mean $A F$ because outliers can distort the mean. As shown in Table 2, the median $A F$ values were proportional to the AUC values of DeepBind models. The sequences generated by our model showed a much higher median $A F$ than random sequences, except for SOX2.

For comparison of our model with AptaSim, we downloaded the HT-SELEX data [19] and ran AptaSim in the AptaSuite collection [20]. The sequences in the first SELEX round of target proteins were used as input to AptaSim. Figure 5 shows the distribution of $A F s$ of the sequences generated by our model, AptaSim and random generator for four target proteins (DRGX, GCM1, OLIG1 and RXRB). The sequences generated by AptaSim showed similar binding affinity as random sequences, but both showed much lower binding affinity than the sequences generated by our model. The nucleic acid sequences used for comparison are available in Additional file 2.

\section{Protein-binding dNA sequence motif}

We generated about 200,000 DNA sequences for NFATC1 using our model, and found a motif (shown in Fig. 6a) conserved in the DNA sequences using DREME [21]. The motif found in the generated DNA sequences was also corroborated by a protein-DNA complex in PDB (Fig. 6d) and known motifs (Figs. 6b and c) from the Homer [22] and JASPAR [23] databases.

In a similar way, we obtained a sequence motif conserved in the DNA sequences for NFKB1 (Fig. 7). DNA sequences and their binding specificity for NFATC1 and NFKB1 are available in Additional file 3.

\section{Comparison with known aptamers}

For comparative purposes of the sequences generated by our model to known aptamers, we selected top 100 DNA sequences with a high binding specificity. We aligned the sequences to each of the known aptamers for NFATC1 [24] and NFKB1 [25] (Additional file 4) using the EMBOSS needleman [26].

As shown in Fig. 8, two alignments of DNA sequences to the NFATC1 aptamer revealed a similar pattern of binding specificity. In the first alignment of DNA sequences to the NFATC1 aptamer, the highest accumulated score of the binding specificity was observed right after the 40-mer region in $5^{\prime}$-GGGAGAGCGGAAGCGUGCUGGGCCN40-CAUAACCCAGAGGUCGAUG GAUCCCCCC-3'. But, in the second alignment the highest score was found in the 40-mer region. These results imply that our approach is useful in finding potential aptamers binding to a target protein. In the alignment, the highest score was observed in the $5^{\prime}$ end of the aptamer, which is a primer site of a random library used when selecting the aptamer. 
Table 2 The median binding affinity AF of the generated sequences and random sequences to target protein $p$ with AUC of DeepBind model of $p$

\begin{tabular}{|c|c|c|c|c|c|c|c|c|}
\hline Protein & DRGX & GCM1 & OLIG1 & RXRB & $\mathrm{SO} 2$ & BHLHE23 & MTF1 & FOXP3 \\
\hline AUC & 0.897 & 0.841 & 0.733 & 0.720 & 0.605 & 0.557 & 0.538 & 0.499 \\
\hline Median AF (Generated) & 0.999 & 0.995 & 0.763 & 0.644 & 0.473 & 0.513 & 0.557 & 0.502 \\
\hline Median AF (Random) & 0.497 & 0.500 & 0.504 & 0.494 & 0.504 & 0.501 & 0.502 & 0.501 \\
\hline
\end{tabular}

We used the model to generate protein-binding RNA sequences as well. As we did for DNA sequences, we trained the model on MBNL1-binding RNA sequences from CLIPdb [27], which were identified by CLIP-seq experiments. We selected top 100 RNA sequences with a high binding specificity (Additional file 3), and aligned them to known MBNL1-binding aptamers [28] (Additional file 4). The known aptamers contain 32-mer MBNL1-binding regions, which are flanked by two constant regions (5'-GGGAAUGGAUCCACAUCUACGAAUUCN32-AAGACUCGAUACGUGACGA ACCU-3').
In both alignments shown in Fig. 9, the highest cumulative score of the binding specificity was observed within the 32-mer MBNL1-binding regions. MBNL1-binding RNAs are known to contain YGCY motifs in their binding regions, where $\mathrm{Y}$ denotes pyrimidine ( $\mathrm{C}$ or $\mathrm{U})$ [28]. It is interesting to note that the motif is observed 3 times (positions 30-33, 41-44 and 47-50) in the 32-mer region of the first alignment, and twice (positions 32-35 and 5053) in the second alignment of Fig. 9. Our model for RNA sequences was trained on data from in vivo experiments (i.e., CLIP-seq), yet generated RNA sequences with similar binding properties as those found by in vitro experiments (i.e., SELEX).
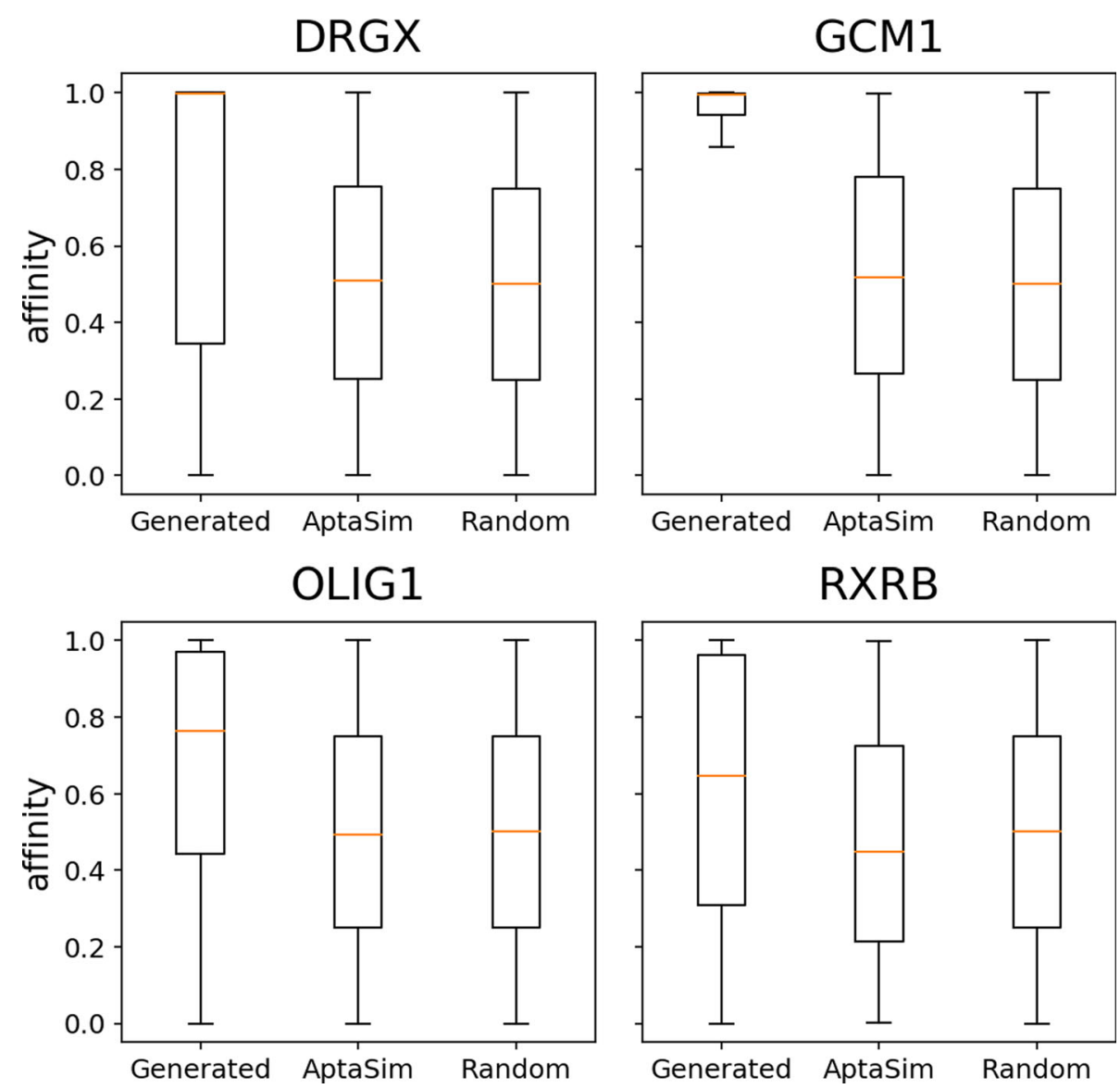

Fig. 5 The binding affinity AF of the nucleic acid sequences generated by our model, AptaSim, and random generator for four target proteins (DRGX, GCM1, OLIG1 and RXRB) 


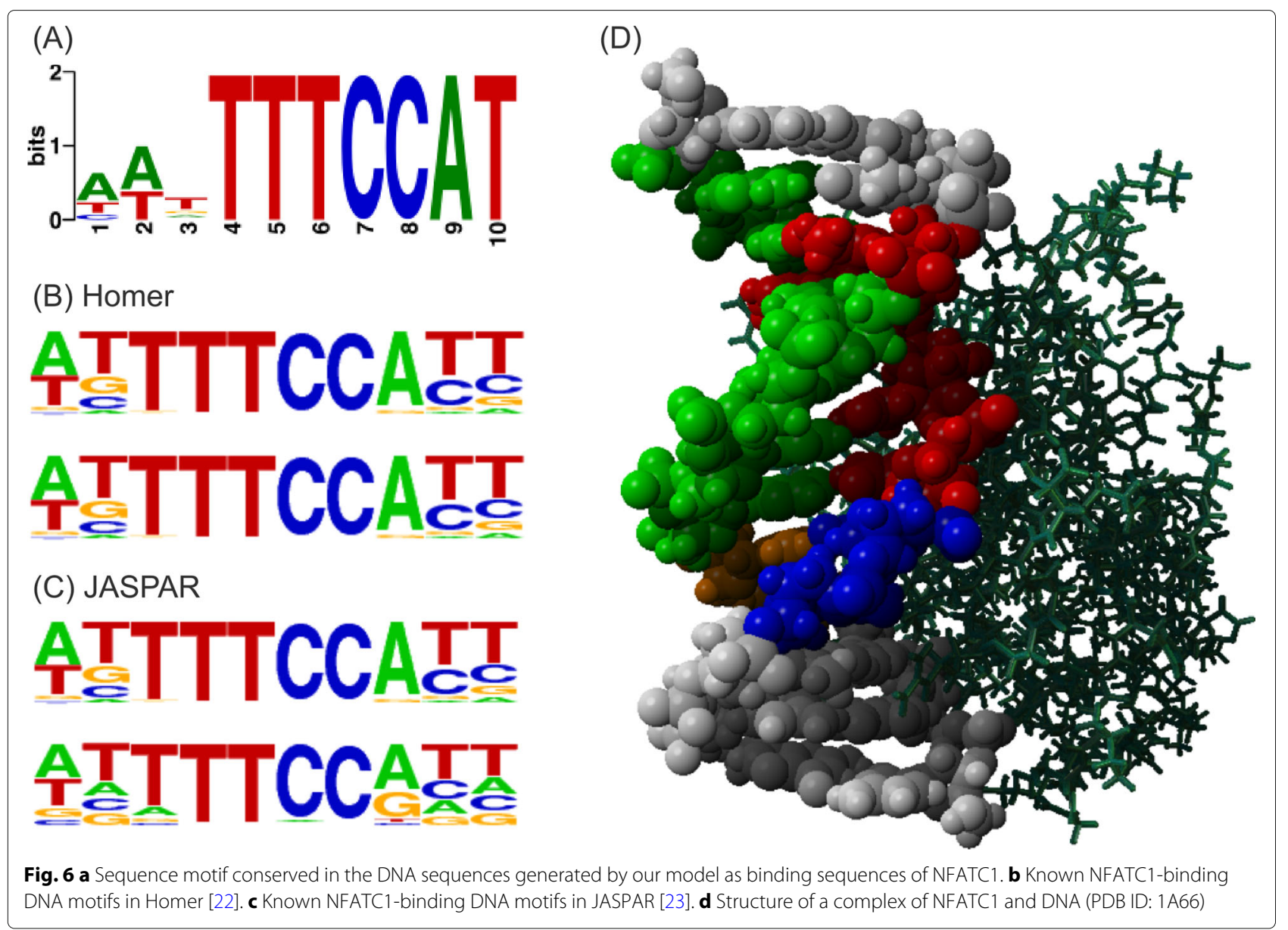




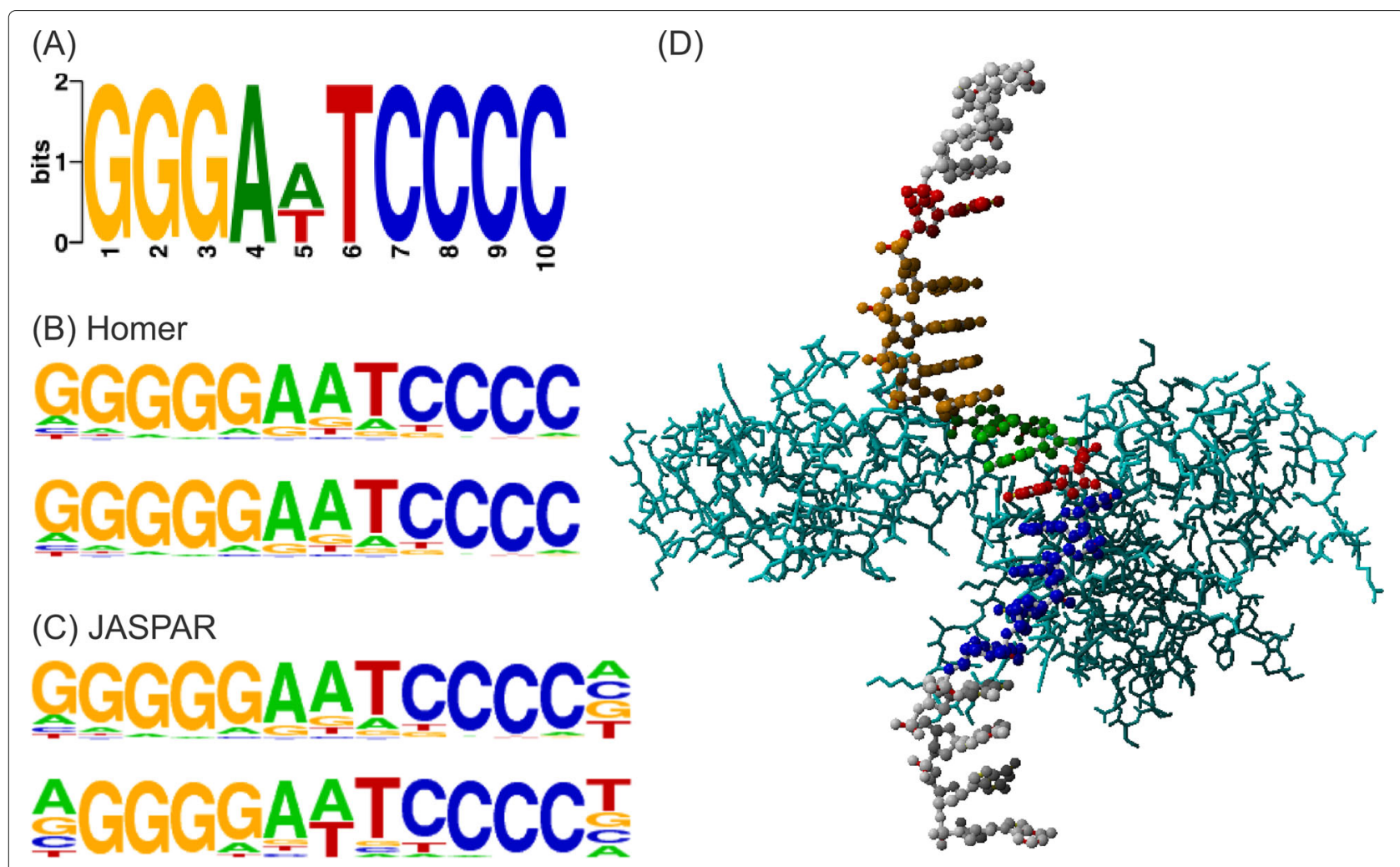

Fig. 7 a Sequence motif conserved in the DNA sequences generated by our model as binding sequences of NFKB1. b Known NFKB1-binding DNA motifs in Homer [22]. c Known NFKB1-binding DNA motifs in JASPAR [23]. d Structure of a complex of NF-kappa B and DNA (PDB ID: 1SVC) 


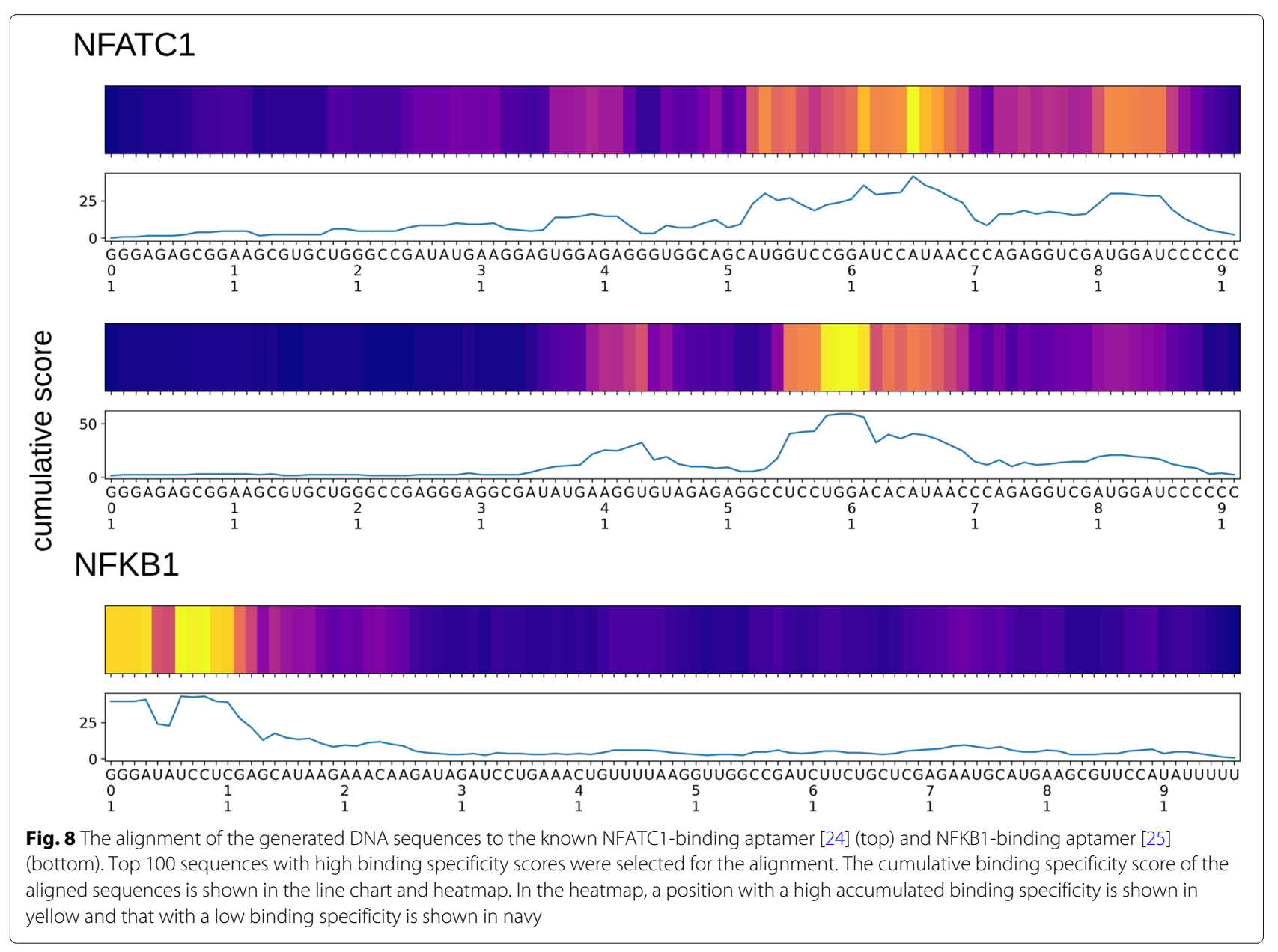




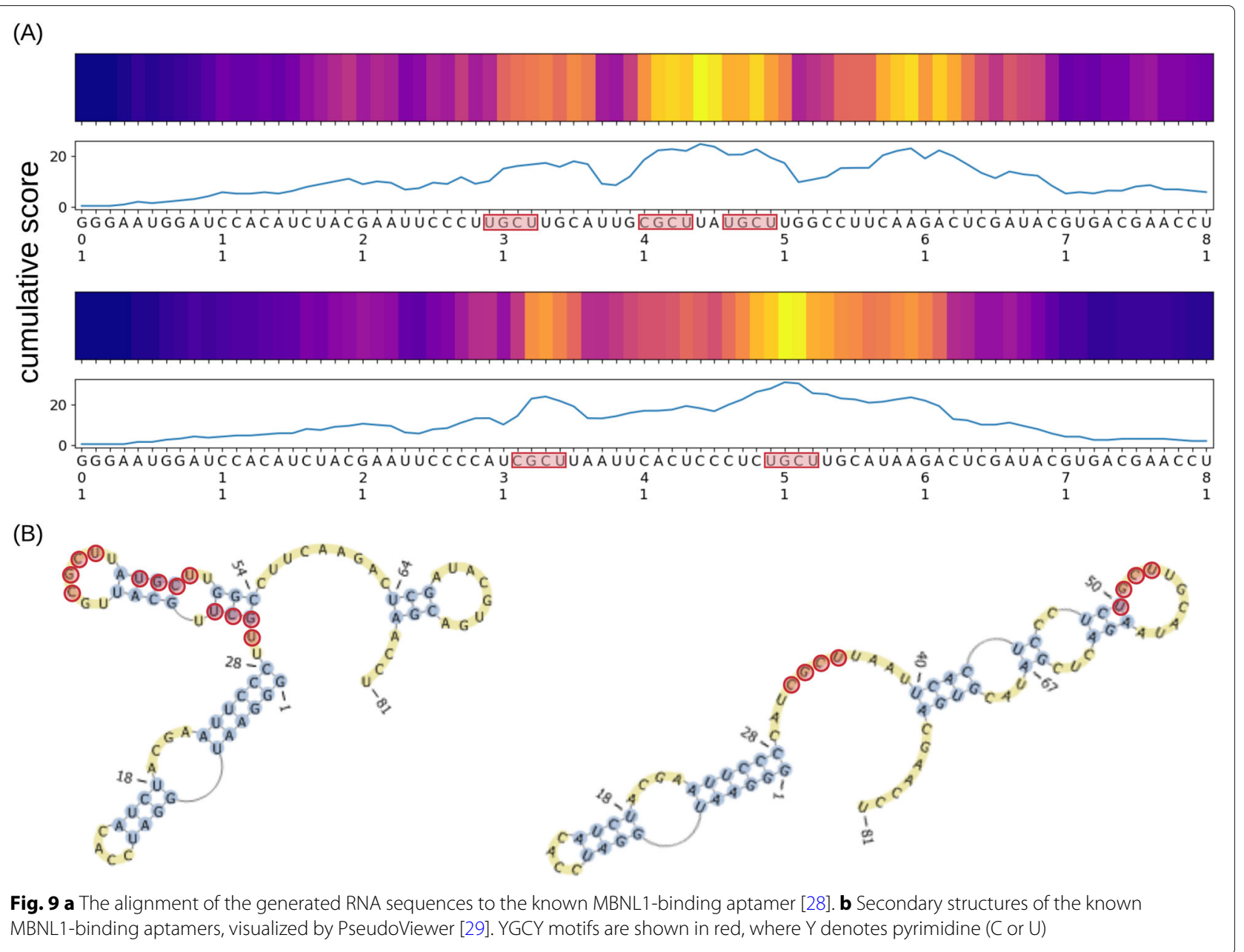

\section{Comparison with other methods}

In "Binding affinity of generated sequences" section and Fig. 5, we compared the binding affinity of the DNA sequences generated by our model with the binding affinity of the DNA sequences generated by AptaSim and random generators. For more extensive comparison, we downloaded HT-SELEX fastq files of NFATC1 and NFKB1 from SRA (https://www.ncbi.nlm.nih.gov/ bioproject/371436), and ran AptaSim. We selected the sequences after 10 rounds and generated motifs conserved in the sequences in the same way that we did for the motifs shown in Figs. 6 and 7. As shown in Additional file 5, the binding motifs conserved in the sequences generated by AptaSim are very different from the well-known binding motifs for NFATC1 (Fig. 6) and NFKB1 (Fig. 7).

For further comparison, we tried a different set of programs in the AptaSuite collection. We first generated a pool of DNA sequences with AptaSim, clustered the sequences with AptaCluster, and found 6- to 10-mer motifs with AptaTRACE. Several motifs were found in the sequences, but the well-known motifs (the NFATC1binding motif TTTCCA and the NFKB1-binding motif GGGGGAATCCCC) were not included in the motifs. Details of the results are available in Additional file 5.

\section{Conclusion}

Many studies have investigated the interactions between nucleic acids and proteins by computational approaches. However, most of the computational approaches treat the problem of nucleic acid-protein interactions as a classification problem. In this paper we proposed a generative model using a recurrent neural network (RNN) to generate nucleic acid sequences binding to a target protein. The model was trained on a huge set of sequences from high-throughput experimental technologies, and tested to construct nucleic acid sequences binding to a target protein. Both DNA and RNA sequences generated by the model for several target proteins showed a high binding specificity, and motifs observed in the sequences were similar to known motifs. 
These are preliminary results of ongoing research, but demonstrated the potential of our approach as a generator of nucleic acid sequences binding to a target protein. In particular, our model will be useful in substantially reducing time and money for in vitro selection of aptamers such as SELEX experiments by constructing an efficient initial pool of nucleic acid sequences.

\section{Supplementary information}

Supplementary information accompanies this paper at

https://doi.org/10.1186/s12864-019-6299-4.

All additional files are available at http://bclab.inha.ac.kr/aptamer.

Additional file 1: HT-SELEX data of DeepBind. The data used for training our generator model and the HT-SELEX data used for comparing with other methods. The size of the compressed file is about 1.8GB.

Additional file 2: Nucleic acid sequences of three types. Nucleic acid sequences generated by our model, AptaSim, and random generator for four proteins (DRGX, GCM1, OLIG1 and RXRB).

Additional file 3: Nucleic acid sequences generated by our model for three proteins. Nucleic acid sequences and their binding specificity to target proteins (NFATC1, NFKB1 and MBNL1), constructed by our model.

Additional file 4: Known aptamers binding to three proteins. An aptamer binding to NFATC1, two aptamers binding to NFKB1, and two aptamers binding to MBNL1.

Additional file 5: FATC1-binding motifs and NFKB1-binding motifs found in the DNA sequences generated by other methods. NFATC1-binding motifs and NFKB1-binding motifs found in the DNA sequences generated by AptaSim and by a set of programs in AptaSuite.

\section{Abbreviations}

AF: Binding affinity; IU: Intersection to union; LSTM: Long short-term memory; RNN: Recurrent neural network; SP: Binding specificity

\section{Acknowledgements}

Not applicable.

\section{About this supplement}

This article has been published as part of BMC Genomics Volume 20 Supplement 13, 2019: Proceedings of the 2018 International Conference on Intelligent Computing (ICIC 2018) and Intelligent Computing and Biomedical Informatics (ICBI) 2018 conference: genomics. The full contents of the supplement are available online at https://bmcgenomics.biomedcentral.com/articles/ supplements/volume-20-supplement-13.

\section{Authors' contributions}

$J$ designed and implemented Sequence generator and prepared the initial manuscript. BP implemented a program for analyzing the results, and helped the initial manuscript. KH supervised the work and wrote the manuscript. All authors read and approved the final manuscript.

\section{Funding}

This work was supported by the National Research Foundation of Korea (NRF) grant funded by the Ministry of Science and ICT (NRF-2017R1E1A1A03069921, NRF-2018K2A9A2A11080914) and the Ministry of Education

(NRF-2016R1A6A3A11931497). Publication of this article was funded by the NRF grant (NRF-2017R1E1A1A03069921).

\section{Availability of data and materials}

Additional files are available at http://bclab.inha.ac.kr/aptamer.

Ethics approval and consent to participate

Not applicable.

\section{Consent for publication}

Not applicable.

\section{Competing interests}

The authors declare that they have no competing interests.

Published: 27 December 2019

\section{References}

1. Walia R, Xue L, Wilkins K, El-Manzalawy Y, Dobbs D, Honavar V. RNABindRPlus: A Predictor that Combines Machine Learning and Sequence Homology-Based Methods to Improve the Reliability of Predicted RNA-Binding Residues in Proteins. Plos ONE. 2014;9(5):. https:// doi.org/10.1371/journal.pone.0097725.

2. Tuvshinjargal N, Lee W, Park B, Han K. PRIdictor: Protein-RNA Interaction predictor. Biosystems. 2016;139:17-22.

3. Choi D, Park B, Chae H, Lee W, Han K. Predicting protein-binding regions in RNA using nucleotide profiles and compositions. Bmc Syst Biol. 2017;11:.

4. Shen Z, Bao WZ, Huang DS. Recurrent Neural Network for Predicting Transcription Factor Binding Sites. Sci Rep. 2018;8:10.

5. Alipanahi B, Delong A, Weirauch M, Frey B. Predicting the sequence specificities of DNA- and RNA-binding proteins by deep learning. Nat Biotechnol. 2015;33(8):831-8.

6. Akbaripour-Elahabad M, Zahiri J, Rafeh R, Eslami M, Azari M. rpiCOOL: A tool for In Silico RNA-protein interaction detection using random forest. J Theor Biol. 2016;402:1-8.

7. Zhang X, Liu S. RBPPred: predicting RNA-binding proteins from sequence using SVM. Bioinformatics. 2017;33(6):854-62.

8. Liu B, Li K, Huang DS, Chou KC. iEnhancer-EL: identifying enhancers and their strength with ensemble learning approach. Bioinformatics. 2018;34: 3835-42.

9. Liu B, Yang F, Huang DS, Chou KC. iPromoter-2L: a two-layer predictor for identifying promoters and their types by multi-window-based PseKNC. Bioinformatics. 2018;34:33-40.

10. Hassanzadeh HR, Wang MD. DeeperBind: Enhancing Prediction of Sequence Specificities of DNA Binding Proteins. In: 2016 IEEE International Conference on Bioinformatics and Biomedicine (BIBM); 2016. p. 178-83. https://doi.org/10.1101/099754.

11. Kim N, Izzo JA, Elmetwaly S, Gan HH, Schlick T. Computational generation and screening of RNA motifs in large nucleotide sequence pools. Nucleic Acids Res. 2010;38(13):e139.

12. Zhou Q, Xia X, Luo Z, Liang H, Shakhnovich E. Searching the Sequence Space for Potent Aptamers Using SELEX in Silico. J Chem Theory Comput. 2015;11(12):5939-46.

13. Hoinka J, Berezhnoy A, Dao P, Sauna ZE, Gilboa E, Przytycka TM. Large scale analysis of the mutational landscape in HT-SELEX improves aptamer discovery. Nucleic Acids Res. 2015;43(12):5699-707.

14. James W. Aptamers. In: Meyers RA, editor. Encyclopedia of Analytical Chemistry. Chichester: Wiley; 2000. p. 4848-71.

15. Hochreiter S, Schmidhuber J. Long short-term memory. Neural Comput. 1997;9(8):1735-80.

16. Graves A, Mohamed A-r, Hinton G. Speech recognition with deep recurrent neural networks. In: IEEE International Conference on Acoustics, Speech and Signal Processing; 2013. p. 6645-9. https://doi.org/10.1109/ icassp.2013.6638947.

17. Sutskever I, Vinyals O, Le QV. Sequence to sequence learning with neural networks. In: Advances in Neural Information Processing Systems 27. Red Hook: Curran Associates, Inc.; 2014. p. 3014-112.

18. Tieleman T, Hinton G. Lecture 6.5-rmsprop: divide the gradient by a running average of its recent magnitude. COURSERA: Neural Networks for Machine Learning, 4; 2012, pp. 26-30.

19. Jolma A, Yan J, Whitington T, Toivonen J, Nitta K, Rastas P, Morgunova E, Enge M, Taipale M, Wei G, et al. DNA-Binding Specificities of Human Transcription Factors. Cell. 2013;152(1-2):327-39.

20. Hoinka J, Backofen R, Przytycka TM. AptaSUITE: A Full-Featured Bioinformatics Framework for the Comprehensive Analysis of Aptamers from HT-SELEX Experiments. Mol Ther Nucleic Acids. 2018;11:515-7.

21. Bailey TL. DREME: motif discovery in transcription factor ChIP-seq data. Bioinformatics. 2011;27:1653-9.

22. Heinz S, Benner C, Spann N, Bertolino E, Lin Y, Laslo P, Cheng J, Murre C, Singh H, Glass C. Simple Combinations of Lineage-Determining Transcription Factors Prime cis-Regulatory Elements Required for Macrophage and B Cell Identities. Mol Cell. 2010;38(4):576-89. 
23. Khan A, Fornes O, Stigliani A, Gheorghe M, Castro-Mondragon J, van der Lee R, Bessy A, Cheneby J, Kulkarni S, Tan G, et al. JASPAR 2018: update of the open-access database of transcription factor binding profiles and its web framework. Nucleic Acids Res. 2018;46(D1):D260-6.

24. Cho J, Lee Y, Shin K, Jeong S, Park J, Lee S. In vitro selection of specific RNA aptamers for the NFAT DNA binding domain. Mol Cells. 2004;18(1): $17-23$.

25. Lebruska $L$, Maher L. Selection and characterization of an RNA decoy for transcription factor NF-kappa B. Biochemistry. 1999;38(10):3168-74.

26. LiW, Cowley A, Uludag M, Gur T, McWilliam H, Squizzato S, Park Y, Buso N, Lopez R. The EMBL-EBI bioinformatics web and programmatic tools framework. Nucleic Acids Res. 2015;43(W1):W580-4.

27. Yang Y, Di C, Hu B, Zhou M, Liu Y, Song N, Li Y, Umetsu J, Lu Z. CLIPdb: a CLIP-seq database for protein-RNA interactions. Bmc Genomics. 2015;16:. https://doi.org/10.1186/s12864-015-1273-2.

28. Goers E, Purcell J, Voelker R, Gates D, Berglund J. MBNL1 binds GC motifs embedded in pyrimidines to regulate alternative splicing. Nucleic Acids Res. 2010;38(7):2467-84.

29. Byun Y, Han K. PseudoViewer3: generating planar drawings of large-scale RNA structures with pseudoknots. Bioinformatics. 2009;25(11):1435-7.

\section{Publisher's Note}

Springer Nature remains neutral with regard to jurisdictional claims in published maps and institutional affiliations.

Ready to submit your research? Choose BMC and benefit from:

- fast, convenient online submission

- thorough peer review by experienced researchers in your field

- rapid publication on acceptance

- support for research data, including large and complex data types

- gold Open Access which fosters wider collaboration and increased citations

- maximum visibility for your research: over $100 \mathrm{M}$ website views per year

At BMC, research is always in progress.

Learn more biomedcentral.com/submissions 